\title{
Nonlinear System Identification based on Orthonormal Wavelet
}

\author{
Luo Xiao ${ }^{1}$, Wu Jianzhen ${ }^{1}$, Li Hongqin ${ }^{1}$ \\ School of Electronic and Electrical Engineering, Shanghai University of Engineering Science, Shanghai ${ }^{1}$
}

\begin{abstract}
Owing to the switching nature of power electronic systems, the dynamic behaviors of these systems are generally nonlinear. It's a good approach to modeling dynamic nonlinear systems using nonlinear system identification. Compactly supported orthogonal wavelets have certain properties useful for system identification. This paper proposed an identification scheme for dynamic nonlinear system model using Haar wavelet. Simulation result for PWM boost DC-DC converter shows the validation of this scheme.
\end{abstract}

Keywords: Wavelet theory, Multi-resolution, Nonlinear system identification.

\section{INTRODUCTION}

The nonlinear systems are widely existed in our world. Owing to the switching nature of power electronic systems, the dynamic behaviors of these systems are generally nonlinear. Many scholars began to use small signal model to establish the dynamic nonlinear model of power electronic system ${ }^{[1]}$. But the small signal model is difficult to deal with large signal disturbance, and can't predict the harmonic and sub harmonic frequency characteristics. In recent years, scholars have put forward a lot of methods of nonlinear modeling of power electronic systems ${ }^{[2]}$. Most of these methods are oriented current, that is, the ideal current requirements, and the system is known to work in detail. In many cases, these conditions are not met, so we consider using the nonlinear identification method to establish the dynamic model. This paper research the nonlinear system identification based on Wavelet.

\section{WAVELET THEORY}

\subsection{Wavelet Transform}

Definition: Suppose $\psi(t) \in L_{2}(R)$, which Fourier transform is $\hat{\psi}(\omega)$. If $\hat{\psi}(\omega)_{\text {satisfied the following }}$ condition:

$$
C_{\psi}=\int_{R}|\hat{\psi}(\omega)|^{2} /|\omega| d \omega<\infty
$$

Then $\psi(t)$ is called Mother Wavelet. The Mather Wavelet could be scaled and shifted as

$$
\psi_{a, b}(t)=a^{-1 / 2} \psi(t-b / a), a, b \in R ; a \neq 0
$$

where is called a Wavelet series. Is the scale factor and is the shift factor.

The continuous Wavelet translation for any is

$$
\begin{aligned}
& f(t) \in L_{2}(R) \text { is } \\
& \quad W_{f}(a, b)=|a|^{-1 / 2} \int_{R} f(t) \psi^{*}(t-b / a) d t
\end{aligned}
$$

and the reconstruction formula (inverse transform ) is

$f(t)=C_{\psi}^{-1} \int_{-\infty}^{\infty} \int_{-\infty}^{\infty} a^{-2} W_{f}(a, b) \psi(t-b / a) d a d b$

Because $\psi_{a, b}(t)$ has the role of the observation window in Wavelet transform for the analyzed signals, $\psi(t)$ must satisfy the constraint condition:

$$
\int_{-\infty}^{\infty} \psi(t) d t<\infty
$$

so $\hat{\psi}(\omega)$ is a continuous function.

The continuous Wavelet must be discretized when utilized in real application. Due to the property of Wavelet basis functions, when parameter ${ }^{a}$ is enlarged, the interval of $a$ is also enlarged at the same time. So let $a=a_{0}^{-j}(j \in Z)$, choose $a_{0}=2$ generally. For the

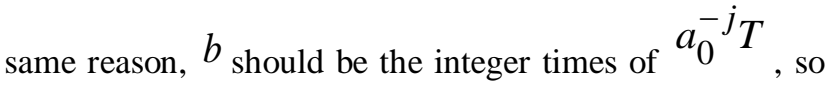
choose $b=k a_{0}^{-j} T$. If $T=1, a_{0}=2$, then $b=k 2^{-j}$. $\psi_{j, k}(t)=a_{0}^{j / 2} \psi\left(a_{0}^{j} t-k\right) \quad$ is the Wavelet basis function with discrete parameters, and the time sampling $\Delta t$ would be $k a_{0}^{-j}$.

For a Wavelet basis function with discrete parameters, the Wavelet coefficients are as following 
Vol. 4, Issue 10, October 2016

$$
C_{j, k}=\int f(t) \psi_{j, k}^{*}(t) d t
$$

and the synthesis or reconstruction for a signal is found $a_{0}, T$ and $\psi(t)$, which satisfied the following equation:

$$
f(t) \approx \sum_{j} \sum_{k} C_{j, k} \psi_{j, k}(t)
$$

2.2 Multi-resolution Analysis and Orthogonal Wavelet Basis

Let $V_{j}$ represents all collections of $L_{2}(R)$ space functions that can be approximated by a $2^{j}$ resolution, $V_{j} \subset L_{2}(R)$

Suppose $\left(V_{j}\right)_{j \in Z}$ is a multi-resolution approximation of $L_{2}(R)$, so a function $\phi(x) \in L_{2}(R)$ is existed (which is called scale function). Let $\phi_{j}(x)=\left.2^{j} \phi\left(2^{j} x\right)\right|_{j \in Z}$, then

$$
\phi_{j, k}(x)=\left.2^{-j / 2} \phi_{j}\left(x-2^{-j} k\right)\right|_{k \in Z}
$$

is a orthogonal basis of $V_{j}$.

Combined with equation $\phi_{j}(x)=\left.2^{j} \phi\left(2^{j} x\right)\right|_{j \in Z}$

equation (8), then the orthogonal basis of $V_{j}$ can be deduced as following

$$
\phi_{j, k}(x)=\left.2^{j / 2} \phi_{j}\left(2^{j} x-k\right)\right|_{k \in Z}
$$

An important feature of the orthogonal wavelet expansion is that the coefficients of the coarse scale can be calculated by the fine scale coefficients recursively and vice versa. For all the resolutions of $f(x)$ (denoted by $\left.2^{j}\right|_{j \in Z}$ ), which approximation can be obtained by the pyramid algorithm.

Above is the case of a single variable. The tensor product method can be used to construct orthogonal basis using $d$ one-dimensional basis functions for multiple variables. Scale function is introduced firstly:

$$
\Phi(x)=\phi\left(x_{1}\right) \times \phi\left(x_{2}\right) \times \cdots \times \phi\left(x_{d}\right)
$$

Substituted $\phi\left(x_{j}\right)$ in equation (10) with $\psi\left(x_{j}\right), 2^{d}-1$ mother wavelets $\Psi^{(i)}(x), i=1, \cdots, 2^{d}-1$ can b obtained[4]:

$$
\begin{aligned}
& \Psi_{j, k}^{(1)}(x)=\psi_{j, k_{1}}\left(x_{1}\right) \phi_{j, k_{2}}\left(x_{2}\right) \cdots \phi_{j, k_{d-1}}\left(x_{d-1}\right) \phi_{j, k_{d}}\left(x_{d}\right) \\
& \Psi_{j, k}^{(2)}(x)=\phi_{j, k_{1}}\left(x_{1}\right) \psi_{j, k_{2}}\left(x_{2}\right) \cdots \phi_{j, k_{d-1}}\left(x_{d-1}\right) \phi_{j, k_{d}}\left(x_{d}\right) \\
& \cdots \\
& \Psi_{j, k}^{(d+1)}(x)=\psi_{j, k_{1}}\left(x_{1}\right) \psi_{j, k_{2}}\left(x_{2}\right) \cdots \phi_{j, k_{d-1}}\left(x_{d-1}\right) \phi_{j, k_{d}}\left(x_{d}\right) \\
& \cdots \\
& \Psi_{j, k}^{\left(2^{d}-1\right)}(x)=\psi_{j, k_{1}}\left(x_{1}\right) \psi_{j, k_{2}}\left(x_{2}\right) \cdots \psi_{j, k_{d-1}}\left(x_{d-1}\right) \psi_{j, k_{d}}\left(x_{d}\right)
\end{aligned}
$$

Then the orthogonal bases of $L_{2}\left(R^{d}\right)$ are

$$
\left\{\Phi_{0 k}(x), \Psi_{j k}^{(1)}(x), \cdots, \Psi_{j k}^{\left(2^{d}-1\right)}(x)\right\} \quad j \in N_{0}, k=\left[k_{1}, \cdots, k_{d}\right] \in Z^{d}
$$

$$
\begin{gathered}
\Phi_{j k}(x)=2^{j d / 2} \Phi\left(2^{j} x_{1}-k_{1}, \cdots, 2^{j} x_{d}-k_{d}\right) \\
\text { where }^{\Psi_{j k}^{(i)}(x)}=2^{j d / 2} \Psi^{(i)}\left(2^{j} x_{1}-k_{1}, \cdots, 2^{j} x_{d}-k_{d}\right) .
\end{gathered}
$$

\section{NONLINEAR SYSTEM IDENTIFICATION BASED ON ORTHOGONAL WAVELET BASES}

The discrete time SISO nonlinear dynamic system is considered:

$$
\begin{aligned}
y(k) & =f[y(k-1), y(k-2), \cdots y(k-p), u(k-1), \cdots u(k-q)] \\
& +C\left(q^{-1}\right) w(k)
\end{aligned}
$$

The input / output series $y(k-i), i=1,2, \cdots, p$ and $u(k-j), j=1,2, \cdots, q$ can be measured, ${ }^{w(k)}$ and $u(k)$ are d uncorrelated zero mean and finite variance measurement noise, whose means $E[w(k)]=0, E[w(k)]^{2}=w, C\left(q^{-1}\right)$ is a stable polynomial about $q^{-1}$.

We can minimize a cost function that is defined by the sum of sampling errors [5]:

$$
J_{K}(\theta)=\frac{1}{K} \sum_{i=1}^{K}\left\|f\left(x_{i}, \omega_{i}\right)-\hat{f}\left(x_{i}, \omega_{i} ; \theta\right)\right\|^{2}
$$

Where $f\left(x_{i}, \omega_{i}\right)$ is the state of next time. $f\left(x_{i}, \omega_{i}\right)$ is known although $f$ is unknown. Suppose $\Psi$ is a compact supported orthogonal wavelet, $\Phi$ is the scale function to generate $V_{j}$ space. According to multi-resolution characteristics,we obtain

$$
\begin{gathered}
V_{j_{0}}=V_{j 1} \dot{+} W_{j 1} \dot{+} W_{j 1+1} \dot{+} \cdots \dot{+} W_{j_{0}-1}, \quad j_{1}<j_{0}-1 \text {, then } \\
f(x)=\sum_{k}\left\langle f, \Phi_{j_{1} k}\right\rangle \Phi_{j_{1} k}(x)+\sum_{j=j_{1} k \in Z^{d}}^{j_{0}} \sum_{l=1}^{2^{d}-1}\left\langle f, \Psi_{j k}^{(l)}\right\rangle \Psi_{j k}^{(l)}(x)
\end{gathered}
$$

Selection of a suitable $j_{1}$ makes the one-dimensional wavelet $\quad \psi_{j_{1}, 0}=2^{j_{1} / 2} \psi\left(2^{j_{1}} x\right)$ and scaling functions 


\section{IJIREEICE \\ International Journal of Innovative Research in Electrical, Electronics, Instrumentation and Control Engineering \\ ISO 3297:2007 Certified \\ Vol. 4, Issue 10, October 2016}

$\phi_{j_{1}, 0}=2^{j_{1} / 2} \phi\left(2^{j_{1}} x\right)$ have small support set, then the sampling distributions in the transforms of $\psi_{j_{1}, k}=2^{j_{1} / 2} \psi\left(2^{j_{1}} x-k\right)$ and $\phi_{j_{1}, k}=2^{j_{1} / 2} \phi\left(2^{j_{1}} x-k\right)$ can be regarded as constant. The first item of equation (14) is the global approximation of $f$ function. So the error of the identification model is

$$
\text { err }=f(x)-\hat{f}(x)
$$

The approximation accuracy can be improved by increasing the $j_{0}$. The coefficients of the newly added basis functions are estimated by using the following formula:

$$
f(x)-\hat{f}(x)=\sum_{k \in Z^{d}} \sum_{l=1}^{2^{d}-1}\left\langle f, \Psi_{j_{0}, k}^{(l)}\right\rangle \Psi_{j_{0}, k}^{(l)}(x)
$$

The new function approach only needs to add new basis functions and their corresponding coefficients on the basis of the existing approximation. Since we choose orthogonal basis, the original basis functions and their coefficients are kept unchanged.

\section{SIMULATION EXAMPLE}

DC-DC converter is a common system in power electronic system ${ }^{[2]}$. We use the boost PWM DC-DC converter as a simulation example (shown as figure 1). he system is composed of a DC voltage source ${ }^{g}$, a constant effect transistor, a diode, an inductor $L$, a capacitor $\mathrm{C}$ and a resistor $\mathrm{R}$. Voltage output is controlled by regulating the switching period of the field effect transistor, so it is called as the working frequency control or pulse width modulation ( PWM ).

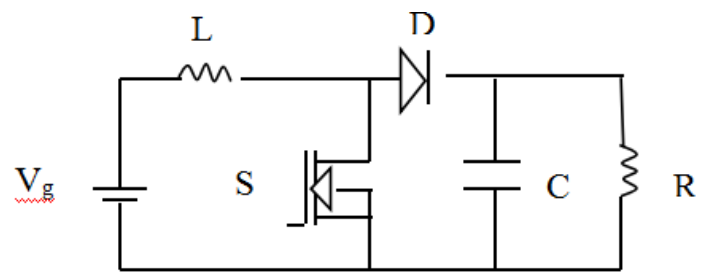

Figure 1 PMW boost converter

We choose Haar wavelet as the basis function in formula (14) in this example. Haar wavelet is not only the earliest usage of wavelet analysis with a close support of the orthogonal wavelet function, but also the simplest function. The definition of Haar wavelet is

$$
\phi(x)= \begin{cases}1 & 0 \leq \mathrm{x}<0.5 \\ -1 & 0.5 \leq \mathrm{x}<1 \\ 0 & \text { others }\end{cases}
$$

The input work cycle is uniformly distributed from 0.35 to 0.65 consistently. 300 sets of data were collected, of which 250 groups were used for identification, and the remaining 50 groups were used for model checking. We choose ${ }^{j_{1}}=1$ and $j_{0}=2$ at the beginning. If the error is too large, we can increase the $j_{0}, d$ is selected as 3 . The Haar wavelet scaling function with the resolution 1 is a zero order spline function with a width of $0.15\left(=\left(0.65-0.35 / 2^{1}\right)\right.$. That is $\phi_{1,0}=\chi([0,0.15))$, the other items in formula (10) are:

$$
\begin{aligned}
\phi_{1, k}= & \chi[0.15 k, 0.15 k+0.15] \\
\psi_{1, k}= & \chi([0.15 k, 0.15 k+0.075])- \\
& \chi([0.15 k+0.075,0.15 k+0.15]) \\
\psi_{2, k}= & \chi([0.075 k, 0.075 k+0.0375])- \\
& \chi([0.075 \mathrm{k}+0.0375,0.075 \mathrm{k}+0.075])
\end{aligned}
$$

Select $k$ coverage to change range from 0.35 to 0.65 , then the basis function in formula (14) is

$$
\left\{\psi_{i}\right\}=\left\{\psi_{1, k}^{(i)}\right\}_{i=1, \cdots, 7, k=2, \cdots, 4} \cup\left\{\psi_{2, k}^{(i)}\right\}_{i=1, \cdots, 7, k=4, \cdots 8} \cup\left\{\phi_{1, k}\right\}_{k=2, \cdots, 4}
$$

So formula (14) can be expressed as

$$
\hat{f}(x)=\sum_{k=2}^{4}\left\langle f, \Phi_{1, k}\right\rangle \Phi_{1, k}(x)+\sum_{j=1}^{2} \sum_{k=2 j}^{4 j} \sum_{l=1}^{2^{3}-1}\left\langle f, \Psi_{j k}^{(l)}\right\rangle \Psi_{j k}^{(l)}(x)
$$

The least square method is used to identify the parameters in the above formula, and the error of the identification model is:

$$
\text { err }=f(x)-\hat{f}(x)
$$

Because the accuracy of the model is not enough, so we increase $^{j_{0}}$, when scale $j_{0}=3$, the coefficients of the newly added basis functions are estimated by using the following formula:

$$
f(x)-\hat{f}(x)=\sum_{k=3}^{12} \sum_{l=1}^{7}\left\langle f, \Psi_{3, k}^{(l)}\right\rangle \Psi_{3, k}^{(l)}(x)
$$

The accuracy of the model is achieved at this time and the simulation result is shown in figure 2. The solid line represents the sample output, and the point line stands for model forecast output. The identification model output of the modeling data is shown in Figure (a) in dotted line while the predictor model output of the validation data is shown in Figure(b) in dotted line. As we can seen from the figure, the model has a good generalization.

\section{CONCLUSIONS}

In order to better research the inherent nonlinear nature of the system, the system identification is an important 


\section{IJIREEICE \\ International Journal of Innovative Research in Electrical, Electronics, Instrumentation and Control Engineering \\ ISO 3297:2007 Certified \\ Vol. 4, Issue 10, October 2016}

method for the modeling of nonlinear systems. In this paper, we study the identification of nonlinear dynamic systems based on orthogonal wavelet bases. Simulation of the boost pulse width modulation DC-DC converter shows that the identification effect of the algorithm is satisfactory. This algorithm does not require a priori knowledge of the system, has high efficiency and good convergence, and can be extended to a large class of nonlinear systems.

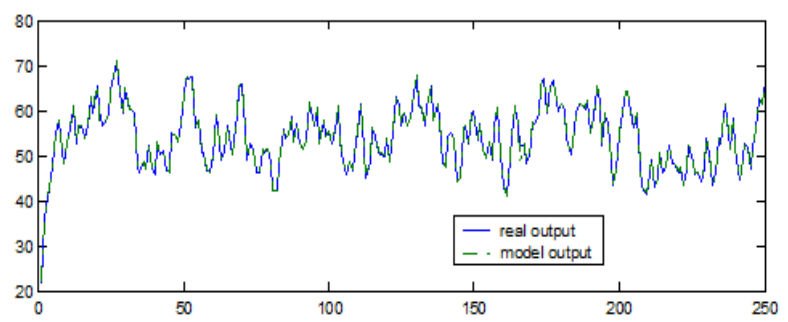

(a) Identification Model

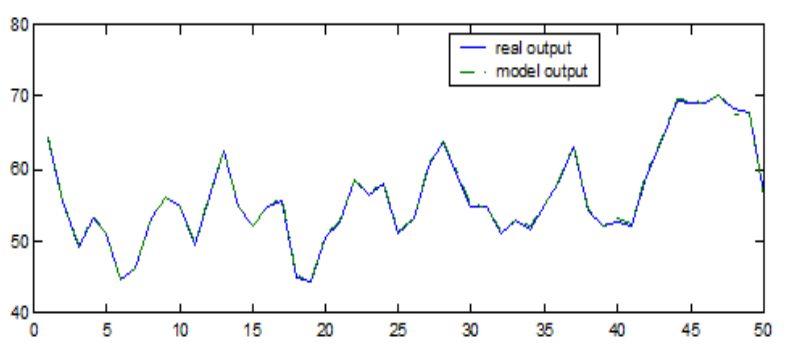

(b) Predictive Model

Figure 2 Simulation result

\section{REFERENCES}

[1] C. C. Chan and K. T. Chau, "Nonlinear modeling of pulsewidthmodulated and quasi-resonant converters," in Proc. Int. Conf. Ind. Electron. Contr. Instru., 1993, pp.361-374

[2] K. T. Chau and C. C. Chan, "Nonlinear identification of power electronic systems," IEEE Trans. Ind. Electron., 1995, Vol. 42, pp. 329-334

[3] Chen Fengshi, "wavelet transform theory and its application in signal processing," the National Defense Industry Press, Beijing, China, 1998

[4] Song Zhihuan, "nonlinear dynamic system identification based on wavelet approximation," Proceedings of the 3th World Congress on Intelligent Control and Automation, 2000, pp. 2226-2229

[5] Sureshbabu, N. and J. A. Farrell, "Wavelet-Based Identification for Nonlinear Control." IEEE Trans. On Automatic Control, Vol. 44 No.2, 1999, pp. 412-417

[6] IA Kougioumtoglou, PD Spanos, "Harmonic Wavelets Based Identification of Nonlinear and Time-Variant Systems". Springer Berlin Heidelberg, 2012, 7520:247-260

[7] P Aadaleesan, N Miglan, R Sharma, P Saha, "Nonlinear system identification using Wiener type Laguerre-Wavelet network model". Chemical Engineering Science, 2008, 63(15): 3932-3941

[8] Śliwiński, Przemysław, "Nonlinear System Identification by Haar Wavelets". Springer Verlag, 2013, 210 